\title{
Game Based Non-Centralized Communication Frequency Choose for OUFS
}

\author{
Jun $\mathrm{Wu}^{1, \text { a }}$, Xiaohao $\mathrm{Mo}^{2, \mathrm{~b},{ }^{*} \text {, Liubin Song }}{ }^{1, \mathrm{c}}$, Bo $\mathrm{Wu}^{1, \mathrm{~d}}$, Litao Chen ${ }^{1, \mathrm{e}}$ \\ ${ }^{1}$ China Communication Technology (Nanjing)Co., Ltd, Nanjing 210007, China \\ ${ }^{2}$ Army Engineering University, Nanjing 210007, China \\ awujun@huaxunchina.com.cn, b," $2847349910 @ q q . c o m,{ }^{c}$ songliubin@huaxunchina.com.cn, \\ dwuboyx@gmail.com, e chenlitao@ huaxunchina.com.cn,
}

\begin{abstract}
The article studies the issue of non-centralized communication frequency choose issue in micro base station (MBS) communication system for maximizing satisfaction utility in opportunity unauthorized frequencies system (OUFS). Considering the operative frequency width limit and capacity demand for each micro base station, our work is to maximize the social welfare of the micro base station communication system which is defined as the satisfaction degree minus price paid. We formulate the issue as a heterogeneous communication frequency choose game. The potential function is designed as the social welfare of the micro base station communication system. We design a heterogeneous communication frequency choose algorithm which asymptotically converges to global optimal point. Simulation results verify our theoretic analysis and the effectiveness of the presented algorithm.
\end{abstract}

Keywords: Communication frequency choose; micro base station; OUFS, game, social welfare.

\section{Introduction}

In $5 \mathrm{G}$ communication, micro base station (MBS) is presented to boost communication system capacity by reusing spectrum more densely. With reduced coverage area, MBS can offload high traffic from macro base station (MBS). OUFS technology is initiated as part of OUFS Release 13 to allow users to access both authorized and unauthorized frequencies under a unified OUFS communication system infrastructure [1]. To utilize the unauthorized spectrum, cellular communication system and wireless devices have to comply with the FCC regulation requirements [2]. OUFS is going to be naturally deployed for micro base stations. Authorized spectrum for micro base station (provisioning the QoS guarantee) can be MBSheduled by the MBS, while unauthorized spectrum is shared among multiple existing technologies or multiple operators. Usually, small base station maybe deployed by individuals and the MBS communication system evolves as a selforganized communication system. Thus, it is hard for MBS to implement resource allocation in unauthorized frequencies for MBSs. A non-centralized approach is more appropriate for MBS to utilize the unauthorized spectrum resource effectively. Unauthorized spectrum has to be carefully sheduled to avoid severe interference.

In this paper, the objective is to maximize the satisfaction utility of MBS communication system. Game theory is introduced to model the interaction among MBSs. We model the non-centralized communication frequency choose issue in both authorized and unauthorized frequencies for MBS communication system as a Task Oriented local interactive heterogeneous communication frequency choose game. MBS acts as a player which conducts communication frequency choose independently. We present a heterogeneous communication frequency choose algorithm for players in the game. Given the presented algorithm, MBSs can learn their actions and converge to the optimal action profile which maximizes the communication system satisfaction utility.

\section{Related Work}

As wireless devices increase dramatically, it becomes harder and unaffordable for MBS to perform resource allocation centrally. It motivates the need for developing autonomous, non-centralized and flexible mobile communication systems where devices can make a decision independently. Usually, 
wireless device's decision in utilizing spectrum may be coupled with others' decisions. The interactive relationship among these spectrum-utilizing devices has to be characterized and game theory emerges as an effective tool. Recently, game theory has been applied extensively to tackle with the wireless resource allocation issue. An interference mitigation game is presented for the noncentralized communication frequency choose in presence of time varying communication frequency in [3]. At least one pure strategy Nash equilibrium (NE) is proved to exist in the game. A completely non-centralized and uncoupled learning algorithm is presented for the player in the game. When all players adhere to the algorithm, game can converge to the pure strategy NE. [4] focuses on a similar topic with [3] but aims to maximize the system throughput. [5] considers a communication system where multiple wireless service providers (WSP) coexist. When concept of OUFS is put forward, both academics and industry show their great interest in it. [6] [8] show their concerns on coexistence of OUFS and $\mathrm{WiFi}$ in the unauthorized spectrum. Analytical results demonstrate that WiFi performance is vulnerable to OUFS interference. Multiple coexistence mechanisms including static muting, listen before talk, self-CTS, and RTS/CTS can be adopted to protect WiFi-only user's service quality. [2] considers micro base station has both cellular interface and WiFi interface. The unauthorized spectrum can be used by WiFi-only user with higher priority. With quality of service (QoS) constraint, resource allocation in both authorized and unauthorized frequencies is constructed as a linear programming issue. In [9], a dual-frequency femtocell (DBF) and a WLAN station are deployed for smart device and WiFi-only device respectively. The unauthorized spectrum is shared between femtocell and WLAN in time.

\section{Interactive Heterogeneous Communication Frequency Choose Game}

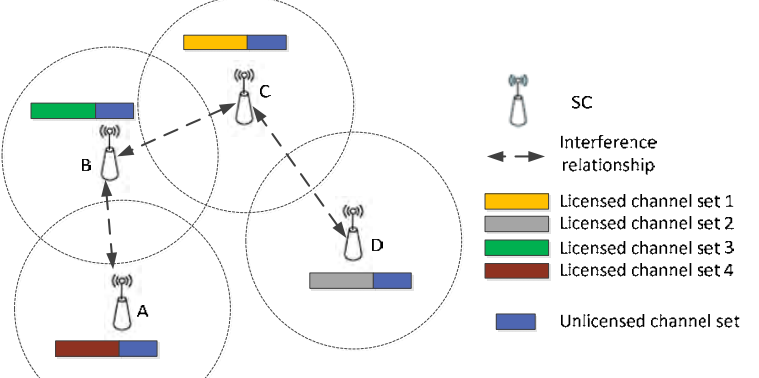

Fig.1 OUFS model

The system model in our work is depicted in figure 1 . The cellular communication system consists of $M$ micro base stations (MBS) which can access both authorized and unauthorized frequencies. Denote the set of MBS as $\mathbf{M}$,i.e. $\mathbf{M}=\{1,2, \cdots, M\}$. Each MBS tries to offload traffic for macro base station (MBS) and MBS decides which communication frequencys in authorized frequencies are available for MBS. We assume each MBS operates with a frequency width limit as $N_{B}$. Though authorized frequencies can provide sustainable quality of service (QoS) for users, MBS has to pay for it. Unauthorized frequency is free, but it has to be shared among MBSs.

Denote $\mathbf{N}_{\mathbf{m}}$ as the available communication frequency set of micro base station (MBS) $m \in \mathbf{M}$, i.e.,

$$
\mathbf{N}_{\mathrm{m}}=\left\{\mathbf{N}_{\mathbf{L}, \mathrm{m}}, \mathbf{N}_{\mathrm{U}}\right\}
$$

$\mathbf{N}_{\mathrm{L}, \mathrm{m}}$ indicates the exclusive authorized communication frequency set for MBS $m$ and $\left|\mathbf{N}_{\mathbf{L}, \mathbf{m}}\right|=N_{L}, m \in \mathbf{M} .|A|$ is the cardinality of $\operatorname{set} A . \mathbf{N}_{\mathbf{U}}$ is the unauthorized communication frequency set. The action set of MBS $m$ can be given as

$$
\mathbf{A}_{\mathbf{m}}=\left\{\mathbf{C}_{\mathbf{L}, \mathbf{m}}, \mathbf{C}_{\mathbf{U}, \mathbf{m}}:\left|\mathbf{C}_{\mathbf{L}, \mathbf{m}}\right|+\left|\mathbf{C}_{\mathbf{U}, \mathbf{m}}\right| \leq N_{B}, \mathbf{C}_{\mathbf{L}, \mathbf{m}} \in \mathbf{N}_{\mathbf{L}, \mathbf{m}}, \mathbf{C}_{\mathbf{U}, \mathbf{m}} \in \mathbf{N}_{\mathbf{U}}\right\}
$$


$\mathbf{C}_{\mathrm{L}, \mathrm{m}}$ and $\mathbf{C}_{\mathrm{U}, \mathrm{m}}$ represent the authorized communication frequency subset and unauthorized communication frequency subset utilized by MBS $m$ respectively. The action set of MBS $m$ can be rewritten as

$$
\mathbf{A}_{\mathbf{m}}=\left\{n_{L, m}, \mathbf{C}_{\mathbf{U}, \mathbf{m}}: n_{L, m} \leq N_{L}, n_{L, m}+\left|\mathbf{C}_{\mathbf{U}, \mathbf{m}}\right| \leq N_{B}, \mathbf{C}_{\mathbf{U}, \mathbf{m}} \in \mathbf{N}_{\mathbf{U}}\right\}
$$

where $n_{L, m}$ is the number of selected authorized communication frequency's and $n_{L, m}=\left|\mathbf{C}_{L, m}\right|$. Suppose MBS $m$ chooses an action $a_{m}=\left\{n_{L, m}, \mathbf{C}_{\mathbf{U}, \mathbf{m}}\right\} \in \mathbf{A}_{\mathbf{m}}$ for communication. We denote capacity for each communication frequency in authorized or unauthorized frequencies as $\alpha$. Then MBS $m$ can obtain its capacity in authorized frequencies as

$$
r_{L, m}=\alpha n_{L, m}
$$

MBS $m$ can deserve its capacity in unauthorized frequencies as

$$
\left.r_{U, m}=\alpha \sum_{i=1}^{n_{U, m}}\left[p(1-p)^{f\left(\mathbf{C}_{\mathrm{U}, \mathbf{m}}(i), a_{J_{m}}\right.}\right)\right]^{\operatorname{sgn}\left(f\left(\mathbf{C}_{\mathbf{U}, \mathbf{m}}(i), a_{J_{m}}\right)\right)}
$$

where $n_{U, m}=\left|\mathbf{C}_{\mathbf{U}, \mathbf{m}}\right|$ and $\mathbf{C}_{\mathbf{U}, \mathbf{m}}(i)$ is the ith communication frequency in set $\mathbf{C}_{\mathbf{U}, \mathbf{m}} \cdot J_{m}$ is the neighbor set of MBS $m$ and $a_{J_{m}}=\left\{a_{k}\right\}, k \in J_{m}$ represents the action profile for MBSs in $J_{m}$. $f\left(\mathbf{C}_{\mathrm{U}, \mathrm{m}}(i), a_{J_{m}}\right)$ indicates the number of MBSs which compete with MBS $m$ for communication frequency $\mathbf{C}_{\mathbf{U}, \mathbf{m}}(i)$ and is defined as

$$
\begin{aligned}
& f\left(\mathbf{C}_{\mathbf{U}, \mathbf{m}}(i), a_{J_{m}}\right)=\sum_{k \in J_{m}} \delta\left(\mathbf{C}_{\mathbf{U}, \mathbf{m}}(i), a_{k}\right) \\
& \delta\left(\mathbf{C}_{\mathbf{U}, \mathbf{m}}(i), a_{k}\right)= \begin{cases}1 & \mathbf{C}_{\mathbf{U}, \mathbf{m}}(i) \in a_{k} \\
0 & \mathbf{C}_{\mathbf{U}, \mathbf{m}}(i) \notin a_{k}\end{cases}
\end{aligned}
$$

$\operatorname{sgn}(\cdot)$ is defined as follow

$$
\operatorname{sgn}(x)=\left\{\begin{array}{l}
1, x>0 \\
0, x=0 \\
-1, x<0
\end{array}\right.
$$

Generally, capacity acquired by MBS $m$ with action $a_{m}$ is given by

$$
c_{m}\left(a_{m}, a_{J_{m}}\right)=r_{L, m}+r_{U, m}
$$

To capture the heterogeneous demand for MBSs, we use a unified Task Oriented utility function to measure the satisfaction of MBSs with different demads which is defined as

$$
f(x)=1-\exp \left(-\frac{\delta x}{d}\right)
$$


$x$ indicates the resource assigned to MBS, $d$ is capacity demand and $\delta$ is an adjustable factor. Combine with (9), the satisfaction degree for MBS $m$ with action $a_{m}=\left\{n_{L, m}, \mathbf{C}_{\mathrm{U}, \mathrm{m}}\right\}$ can be derived as

$$
S d_{m}\left(a_{m}, a_{J_{m}}\right)=f\left(c_{m}\left(a_{m}, a_{J_{m}}\right)\right)=1-\exp \left(-\frac{\delta c_{m}\left(a_{m}, a_{J_{m}}\right)}{d_{m}}\right)
$$

where $d_{m}$ is the capacity demand for MBS $m$.

With the consideration of price paid for authorized communication frequencys, we design the satisfaction utility for MBS $m$ as

$$
g_{m}\left(a_{m}, a_{J_{m}}\right)=S d_{m}\left(a_{m}, a_{J_{m}}\right)-n_{L, m} \beta
$$

where $\beta$ is the price paid for each authorized communication frequency.

Accordingly, the micro base station communication system satisfaction utility can be defined as

$$
U_{0}=\sum_{m \in \mathbf{M}} g_{m}\left(a_{m}, a_{J_{m}}\right)
$$

Then, the global objective is to find the optimal action profile to maximize the communication system satisfaction utility, i.e.,

$$
(P): \max U_{0}
$$

The presented game is denoted as $\mathcal{G}=\left[\mathbf{M},\left\{\mathbf{A}_{\mathbf{m}}\right\}_{m \in \mathcal{M}},\left\{J_{m}\right\}_{m \in \mathbf{M}},\left\{U_{m}\right\}_{m \in \mathbf{M}}\right]$, where $\mathbf{M}$ is the set of players (MBS), $\mathbf{A}_{\mathbf{m}}$ is the set of available actions for player $m, J_{m}$ denotes set of neighbors of player $m$ and $U_{m}$ is the utility function of player $m$. In this paper, we define the utility function of MBS $m$ as

$$
U_{m}\left(a_{m}, a_{J_{m}}\right)=g_{m}\left(a_{m}, a_{J_{m}}\right)+\sum_{k \in J_{m}} g_{k}\left(a_{k}, a_{J_{k}}\right)
$$

\section{Heterogeneous Communication Frequency Choose Algorithm}

To maximize the communication system utility, a heterogeneous communication frequency choose algorithm is presented for MBSs in the game. The procedure of the algorithm is depicted as follows.

\section{Simulation Results and Discussion}

In the simulation, we consider a communication system consists of $6 \mathrm{MBSs}$ which are deployed densly or sparsly. We call any two MBSs neighbors when they locate in the other's interference region. For each authorized or unauthorized communication frequency, $\alpha=4 M b p s$ can be supported. We note that the operating frequency width limit and the reserved authorized communication frequencys for each MBS are given as $B=3$ and $N_{L}=3$ respectively. MBS will transmit on the unauthorized communication frequency which is also utilized by other MBSs with probability $p=0.5$. In the unified Task Oriented utility function, $\delta=-3$ is fixed. According to the experimental results, the learning factor in the DAHCS algorithm is given as $\gamma=i$ in the $i$ th iteration. 


\section{Algorithm 1: Heterogeneous Communication frequency Choose Algorithm}

Step 1. Initially, set $k=0$ and let each MBS $m \in \mathbf{M I}$ selects an action $a_{m}(0)$ from its available action set $A_{m}$ with equal probability.

Step 2. MBSs exchange the information with their neighbors.

Step 3. A MBS $i$ is selected randomly from M. All other MBSs will repeat their actions as $a_{-i}(k+1)=a_{-i}(k)$. And for MBS $i$, we will decide the probability distribution of choose for action $a_{i}(k+1)$. If MBS $i$ selects action $a_{t}(k+1)=a_{i}$, it will obtain its utility as $U_{i}\left(a_{i}, a_{J_{i}}(k)\right)$ according to the information from neighbors. Then, we could acquire the probability for selecting $a_{i}(k+1)=a_{i}$ as follows

$$
p_{i}^{a}(k+1)=\frac{\exp \left\{\gamma U_{i}\left(a_{i}, a_{J_{i}}(k)\right)\right\}}{\sum_{a_{e}, \mathcal{A}_{i}} \exp \left\{\gamma U_{i}\left(\bar{a}_{i}, a_{J_{i}}(k)\right)\right\}}
$$

Where $\gamma$ is a learning factor. $\sum_{\bar{a} \in \lambda_{i}} \exp \left\{\gamma U_{i}\left(\bar{a}_{i}, a_{J_{i}}(k)\right)\right\}$ represents the aggregate utility for

MBS $i$ performing all actions in $\mathbf{A}_{i}$. Then, MBS $i$ decides its action $a_{i}(k+1)$ with the probability distribution $\left\{p_{t}^{q}(k+1)\right\}_{a \in d}$.

Step 4. If the predefined maximum number if iteration is achieved, stop; else go to Step 2.

Fig.2 untility comparison result

In figure 3, we compare the performance in communication system satisfaction utility among methods below: exhaust searching (ES), DAHCS algorithm and random approach. In the simulation, $N_{U}=2$ unauthorized communication frequencies are available and price for licnesed communication frequency is normalized as $\beta=0.2$. We also set $\mathbf{D}=\{10 \mathrm{Mbps}, 6 \mathrm{Mbps}, 8 \mathrm{Mbps}, 11 \mathrm{Mbps}, 5 \mathrm{Mbps}, 9 \mathrm{Mbps}\}$ to capture the heterogeneous demand of different MBSs. By exhaust searching, each MBS can acquire its optimal action to maximize communication system satisfaction utility. However, a central controller is needed to MBShedule each MBS's action choose. Moreover, as number of MBSs increases, the computation complexity will grow rapidly. Unlikely, with DAHCS algorithm MBS can learn its action by exchanging information with its neighbors and make a decision automatically in the presented game framework. By learning, MBSs can also achieve the optimal action profile distributly which is proved in the previous section and validated in the simulation. As shown in the figure, the game plays 200 times and converges aound $40^{\text {th }}$ iteration. The maximum communication system satisfaction utility is then acuqired with the optimal action profile adopted by all MBSs. While under the random approach, each MBS randomly select an action for itself per iteration regardless of the potential interference and capacity demand. Therefore, as shown in the figure a favorable performance cannot be guaranteed . 


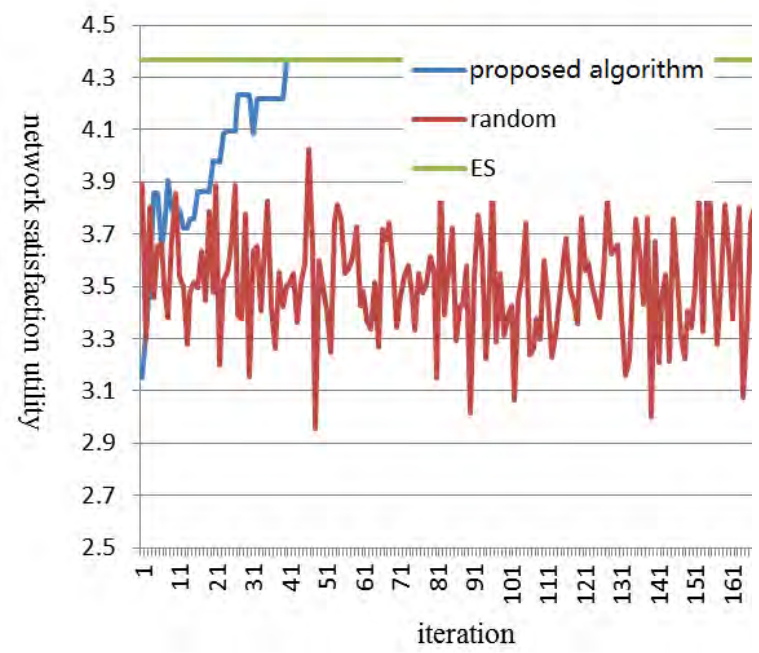

Fig.3 untility comparison result

\section{Conclusion}

In our work, we consider OUFS is deployed for micro base station. Then micro base station can access both authorized and unauthorized frequencies. Micro base station has to pay for authorized frequencies which are for exclusive use. While unauthorized spectrum is free for micro base stations but may be shared with others. Taking the operating frequencywidth limit and capacity demand into consideration, we investigate the non-centralized communication frequency choose issue in authorized and unauthorized frequencies to maximize the aggregate satisfaction utility in the micro base station communication system. The interactive heterogeneous communication frequency choose game is presented to depict the optimization issue. At last, we present the heterogeneous communication frequency choose algotithm, adhere to which the game can converge to the optimal action profile with an arbitrarily high probability. The simulation results validate the efficiency of the algorithm.

\section{References}

[1]. Rupasinghe, N.; Guvenc, I., "Authorized-assisted access for WiFi-OUFS coexistence in the unauthorized spectrum," Globecom Workshops (GCWkshps), 2014, vol., no., pp.894,899, 8-12 Dec. 2014.

[2]. Elsherif, A.R.; Wei-Peng Chen; Ito, A.; Zhi Ding, "Adaptive micro base station access of authorized and unauthorized frequencies," communications (ICC), 2013 IEEE International Conference on, vol., no., pp.6327,6332, 9-13 June 2013.

[3]. Qihui Wu; Yuhua Xu; Jinlong Wang; Liang Shen; Jianchao Zheng; Anpalagan, A., "Noncentralized Communication frequency Choose in Time-Varying Radio Environment: Interference Mitigation Game with Uncoupled Stochastic Learning," in Vehicular Technology, IEEE Transactions on, vol.62, no.9, pp.4524-4538, Nov. 2013.

[4]. Yuhua Xu; Jinlong Wang; Qihui Wu; Anpalagan, A.; Yu-Dong Yao, "Opportunistic Spectrum Access in Unknown Dynamic Environment: A Game-Theoretic Stochastic Learning Solution," in Wireless Communications, IEEE Transactions on, vol.11, no.4, pp.1380-1391, April 2012.

[5]. Feng Zhang; Wenyi Zhang; Qiang Ling, "Non-Cooperative Game for Capacity Offload," in Wireless Communications, IEEE Transactions on, vol.11, no.4, pp.1565-1575, April 2012.

[6]. Jeongho Jeon; Li, Q.C.; Huaning Niu; Papathanassiou, A.; Geng Wu, "OUFS in the unauthorized spectrum: A novel coexistence analysis with WLAN systems," Global Communications Conference (GLOBECOM), 2014 IEEE, vol., no., pp.3459,3464, 8-12 Dec. 2014. 
[7]. Cavalcante, A.M.; Almeida, E.; Vieira, R.D.; Chaves, F.; Paiva, R.C.D.; Abinader, F.; Choudhury, S.; Tuomaala, E.; Doppler, K., "Performance Evaluation of OUFS and Wi-Fi Coexistence in Unauthorized Frequencies," Vehicular Technology Conference (VTC Spring), 2013 IEEE 77th, vol., no., pp.1,6, 2-5 June 2013.

[8]. Jeongho Jeon; Huaning Niu; Li, Q.C.; Papathanassiou, A.; Geng Wu, "OUFS in the unauthorized spectrum: Evaluating coexistence mechanisms," Globecom Workshops (GC Wkshps), 2014, vol., no., pp.740,745, 8-12 Dec. 2014.

[9]. Liu, F.; Bala, E.; Erkip, E.; Beluri, M.; Yang, R., "Micro base station Traffic Balancing Over Authorized and Unauthorized Frequencies," in Vehicular Technology, IEEE Transactions on, vol. PP, no.99, pp.1-1, 2015. 